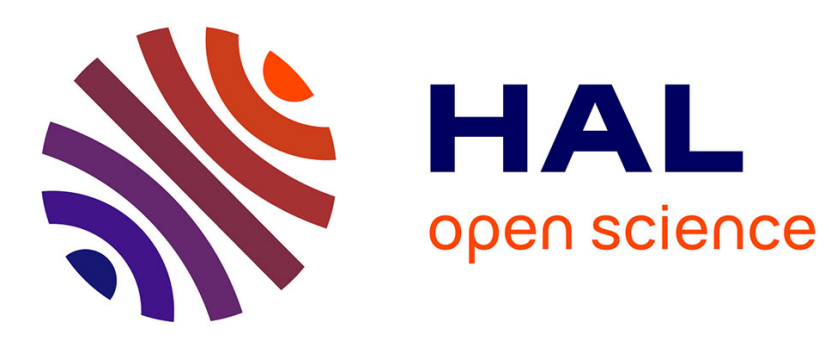

\title{
Specificity of pharmacokinetic modeling of nanomedicines
}

Vincent Lebreton, Samuel Legeay, Patrick Saulnier, Frederic Lagarce

\section{To cite this version:}

Vincent Lebreton, Samuel Legeay, Patrick Saulnier, Frederic Lagarce. Specificity of pharmacokinetic modeling of nanomedicines. Drug Discovery Today, 2021, 10.1016/j.drudis.2021.04.017 . hal03207647

\section{HAL Id: hal-03207647 https://hal.science/hal-03207647}

Submitted on 26 Apr 2021

HAL is a multi-disciplinary open access archive for the deposit and dissemination of scientific research documents, whether they are published or not. The documents may come from teaching and research institutions in France or abroad, or from public or private research centers.
L'archive ouverte pluridisciplinaire HAL, est destinée au dépôt et à la diffusion de documents scientifiques de niveau recherche, publiés ou non, émanant des établissements d'enseignement et de recherche français ou étrangers, des laboratoires publics ou privés. 


\title{
Specificity of pharmacokinetic modeling of nanomedicines
}

Vincent Lebreton ${ }^{1,2}$, Samuel Legeay $^{1}$, Patrick Saulnier ${ }^{1,2}$, Frederic Lagarce ${ }^{*^{1,2}}$

1: University of Angers, MINT Inserm 1066 CNRS 6021, Angers, France

2: CHU Angers, 4 rue Larrey, 49033, Angers, France

$(*)$ : corresponding author Frederic.lagarce@univ-angers.fr ; Angers, France

Tel: +33241353544

\section{$\underline{\text { Teaser }}$}

This review discuss why regular pharmacokinetic modeling is not relevant for nanomedicines in many cases and how it impedes the market access of those drugs.

\section{Keywords}

Nanomedicine, pharmacokinetics, drug evaluation, modeling

\begin{abstract}
Nanomedicines have been developed for more than 4 decades in order to optimize the pharmacokinetics of drugs, especially absorption, distribution and stability in vivo. Unfortunately, only a few drug products reached the market. One reason among others is the lack of proper pharmacokinetic modeling and evaluation that impedes the optimization of those promising drug delivery systems. In this review the specificity of nanomedicines are discussed. Key parameters to take into account for future accurate pharmacokinetic evaluation of nanomedicine are proposed. We believe this could help those innovative drug products to reach to market and change the fate of many diseases.
\end{abstract}

Published in Drug discovery Today, 2021 Apr 20:S1359-6446(21)00201-4.

doi: 10.1016/j.drudis.2021.04.017

PMID: 33892140 


\section{Introduction}

The concept of nanomedicines has been coined in the 1960 s by a few pioneers. After 60 years, some successful medical applications can be found in the market especially in the domain of cancer therapy and recently with the two nanoparticle (NP)-based vaccines to combat COVID-19 [1-4]. However, for some key opinion leaders in this field, the pathway to the market is very narrow for those special drug delivery systems [5]. This issue is linked to the difficulty to well characterize the systems in the nanosize range [6]. The clinical translation would be faster if the products were better characterized and if their behavior in vivo was more described [7].

The game is worth the candle because nanotechnologies have some great potentials in medicine beyond their application for vaccines. In the beginning, this concept of nanodrug delivery system was proposed to improve the efficacy and safety of already commercialized anticancer or anti-infectious drugs. The objective was to enhance the performance of those nano-encapsulated drugs (absorption, body residence time, selective distribution) and to limit their toxicity [8]. This field has received a lot of funding for more than 20 years and is still projected to grow rapidly [9].

Unfortunately, the few nanoformulations in therapeutics that have already reached the market did not dramatically change the outcome of their target disease. This is, for example, the case for liposomal doxorubicin which displays a new toxicological profile in comparison to free doxorubicin (palmoplantar erythrodysesthesia)[10], [11] likewise for $\mathrm{Nab}^{\circledR}$ paclitaxel (increase sensory neuropathy) which has not changed the prognosis of breast cancer tumor [11-12]. The enhanced permeation and retention effect observed in rodents, which was for decades used to explain the potential of NP to accumulate into tumors, is now widely recognized as minimal in humans [13]. These few examples show that the biodistribution of nanomedicines remains to be precisely determined. Moreover, the interactions of nanomedicines with biological media needs to be more described [14], in particular, because of the high surface to mass ratio of those nanosized drug delivery systems. Indeed, one major interaction raised to explain the fate of NP in vivo is the protein corona formation at the surface of the NP, the diffusion of the modified NP being impeded by its sterically hindered surface.

We think that the major problem impeding the access of nanomedicine to patients is that previous pharmacokinetic (PK) and biodistribution studies, including PK modeling, did not take into account the specificity of those drug delivery systems. In regular PK analysis, biodistribution is described by 4 phases: absorption (except for IV route), distribution, metabolism and elimination. They are associated to different PK parameters: volume of distribution, half-life, mean retention time (MRT), area Under the curve (AUC) and clearance for example. Those parameters are calculated from concentration of the drug molecule in the blood (mainly) versus time. The PK modeling can be done either by a non-compartmental approach or either by compartmental modeling. In the present review, we will try to shed some light on what makes nanomedicine so different and how those specificities should be considered in models that build their approval by health national and international agencies.

Nanomedicines can globally be classified into two groups: organic and inorganic. Organic NPs include nanocapsules, dendrimers, polymeric micelles, and liposomes while inorganic ones include NP without any carbon atom and are generally composed of a variety of materials such as noble metals (golden, silver...), alkaline earth metals (calcium, magnesium...), semiconductors (cadmium, titan) or magnetic compounds (iron, nickel, cobalt...)[15].

This reflection will focus on organic lipidic or polymeric NP as the issues and models would be different from inorganic particles especially considering their diffusion and stability which are central to understand the fate of those particles in the body.

Our discussion will follow the three main stages of PK after administration i.e. Absorption, distribution and elimination. For each stage we will discuss how to take into account the specificity of nanocarriers in view of a relevant PK study.

\section{Absorption}

Besides parenteral routes, all routes of administration must pass through the absorption process to reach the blood circulation for systemic action. This is for example the case for skin delivery, pulmonary delivery, and oral delivery [16]. We will focus our discussion on oral delivery because the challenges for PK evaluation are the highest for this route, as explained below.

The oral route is the route of choice for many drugs because it is considered convenient and safe. However, some drugs are not properly absorbed by the oral route because they are not soluble and display a low permeability across the intestinal barrier. Some drugs are also destroyed by the 
biochemical stress induced by digestive liquids. To protect the drug and enhance its permeability, nanomedicines have been proposed. This would potentially allow the oral delivery of some peptides such as insulin or some other important drugs such as anticancer drugs [17].

After oral administration, the bioavailability of the drug is mainly impacted by the absorption process. Actually, the stability of the drug after contact with biological media, its solubility, and its permeability are key parameters to monitor. The encapsulation of the drug molecules in NP may help to master these parameters and many examples can be found showing oral bioavailability enhancement after encapsulation in nanocarriers [18-21] but specific issues may also appear as we will discuss below.

\section{Gastrointestinal stability of NP in relation to their PK profile}

Gastric or intestinal fluids are well-known stress for free molecules because of the $\mathrm{pH}$ of the media and the presence of enzymes such as lipases or peptidases. If encapsulation is proposed to protect active drugs, NPs should remain intact after contact with gastric or intestinal fluid with a minimal encapsulated drug leakage. To evaluate gastrointestinal stability of lipid nanocapsules (LNC), Roger et al, studied the size of LNC after 3 hours of contact with a simulated gastric (FaSSIFV2) and intestinal media (FeSSIF-V2). The LNCs size was not modified after $3 \mathrm{~h}$ in the gastric medium but quickly destroyed in the intestinal medium because of pancreatic lipase [22]. In addition, Singh et al studied the stability of PEGylated liposomes vs. conventional liposomes after 2 hours of exposure to the harsh conditions of the gastrointestinal environment. They compared the release of the drug from those 2 formulations and observed that if the drug remained in PEGylated liposomes after 2 hours, the drug was totally released with conventional liposomes [23]. For polymeric NP, Tobio et al have also demonstrated that a PEG coating of poly (lactic acid) (PLA) NP increases protection against digestive fluids. Indeed, after 4 hours of incubation in simulated gastric media, PEG- PLA NP were less degraded than PLA NP (only 3\% PEG PLA NP were converted in lactate against $9 \%$ for PLA NP) [24].

From the previous examples it is easy to understand that in order to describe the PK profile of nanomedicines after oral administration, the stability of the nanocarrier along all the gastrointestinal tract should be checked and included in the PK model.

\section{Mucus permeation of NP as a critical barrier for absorption}

After its contact with gastric and intestinal fluid, the nanocarrier has to diffuse through mucus to be absorbed or at least to release its cargo in the mucus in the vicinity of drug absorption sites. After the stability issue, the diffusion within mucus has been raised as an important barrier for nanocarrier absorption and barrier properties of mucus have been fully reviewed elsewhere [25-27].

Groo et al studied the impact of LNC surface modification on their diffusion capability through intestinal mucus layer with Transwell ${ }^{\circledR}$ system. They conclude that LNCs with positive or neutral charge better diffuse through the mucus than negative LNCs [28]. However, mucus diffusion may be a limiting step for drug absorption in the case of nanomedicines, explaining why many authors have proposed specific models [26] and NP surface modifications to ease mucus crossing [29]. Regular cell models such as Caco- 2 that are widely used to assess the intestinal permeability of small molecules are not relevant in the case of nanomedicines if they do not display mucus barrier. This is why specific in vitro models including mucus-secreting cells in co-culture with Caco-2 have been proposed [30]-[32]. For example, to increase the correlation between in vitro/in vivo results, Pereira et al. seeded Caco-2 and HT29-MTX Matrigel $^{\circledR}$ which contains myofibroblasts (CCD18-Co cells)[33]. The authors demonstrated that this new $3 \mathrm{D}$ in vitro model is robust and could be used to predict drug and NP permeability [33].

NP mechanisms of diffusion in mucus are a complex and ill-defined phenomenon. Physicochemical interactions like hydrophobic, electrostatic, and hydrogen interactions limit NP diffusion and can be controlled by the composition of the NP surface properties [26]. In addition, because both the size and the charge of nanomedicines have an impact on their ability to diffuse through mucus and that there is an interplay between those two features, they have to be analyzed concomitantly [34-35].

Contrary to what is used for free small molecules, mucus diffusion is a key parameter that must be considered in the design of absorption models for nanomedicines. Thus, as proposed for stability, relevant models of PK used in the case of nanomedicines should take into account their diffusion into mucus. In the case of small molecules, in vitro/in vivo correlation does not consider mucus diffusion; i.e the more the drug is released in vitro the most it will be absorbed (if the transport process is not saturable). This correlation is much more complicated if the NP are stuck into mucus. Similarly, current PK models do not differ if 
the drug remains in the nanoparticle during the absorption process.

\section{Crossing intestinal barrier: the specificity of nanomedicines and consequences on PK study}

Crossing the intestinal barrier requires specific pathways in the case of nanomedicines. As long as the drug remains encapsulated, it cannot cross the enterocytes by passive diffusion, whereas it is the main absorption process for small uncharged molecules. The absorption will thus be different if the drug is released before enterocyte crossing or if it is the NP carrying the drug that is absorbed. Those differences have to be taken into account in relevant specific PK models. Indeed, NP can only be absorbed by active endocytosis using clathrin pits or caveolae or by micropinocytosis[32]. These processes are saturable and slower than passive diffusion. These particular steps for NP absorption should be included in relevant PK models of NP. Absorption mechanisms can be studied using regular cellular models [18]. For example, one important question to address is the equilibrium of the endocytosis mechanisms in the case of NP. For some NPs, a relay between the different endocytosis has been observed if one of them is saturated or blocked allowing a rapid uptake of the NP in the cell but for others, it may not be the same if their uptake is mediated by only one endocytosis process.

The integrity of NP through their crossing should also be studied. As an example, Roger et al, have conducted an in vitro study of LNCs to demonstrate their stability during enterocyte crossing. By following NP with Förster Resonance Energy Transfer (FRET) labelling method, they observed after 2 hours the FRET signal in the basolateral side of the Caco- 2 cell monolayer, allowing them to demonstrate LNC integrity along with intestinal absorption [36]. This information is essential because the PK after absorption will follow the PK profile of the free drug if the carrier is destroyed during the absorption process, whereas it will mostly follow the PK profile observed after IV injection of the nanocarrier if this carrier remains intact after reaching the portal vein at the end of the absorption process. One can also advance that the PK of the drug can also be a complex mix between the free and the encapsulated drug.

Other in vitro models were developed to accurately mimic the cell diversity of the intestinal barrier. Gulberg et al developed a co-culture with Caco-2 cells and B-cell Lymphoma Raji line to mimic enterocytes and $M$ cells respectively [37]. This model was improved by des Rieux et al., and allowed the authors to suggest micropinocytosis as a main mechanism of absorption for their $200 \mathrm{~nm}$ carboxylated polystyrene NP [30]. Beloqui et al. summarized different cell culture models used for the transport study of NP. She states that almost all NP intestinal crossing used endocytosis except for lipophilic NP, which are mainly transported by transcellular pathway [32].

The cellular models for studying intestinal absorption have been improved in order to better describe their uptake mechanisms by the cells. The knowledge of those mechanisms is interesting to optimize the nanoparticle formulation to enhance their oral bioavailability. In parallel, the integrity of NP can be monitored (especially using FRET) through the crossing of the cells. This second feature seems much more critical to include in the PK model in order to more accurately predict the bioavailability of the drug. If the NP is not stable while crossing the intestinal cell barrier, the fate of the encapsulated drug can be critically modified, which is not the case if the uptake pathway changes or is not totally described. The latter is shown in a paper from Roger et al, displaying alternative uptake mechanisms as the main entrance pathway was blocked by inhibitors. Indeed, they have demonstrated after cholesterol depletion or inhibition of clathrin-mediated endocytosis a modification of transport pathways [21].

\section{PK model relevancy for absorption step}

From what has been already discussed, we can affirm that integrity of the NP and its overall stability is an important parameter to monitor to obtain a relevant PK model. In regular PK models, the AUC after oral administration is the accurate evidence of the rate of absorption that can be calculated by Weibull modeling (Weibull's law is a 3-parameter probability law that is widely used to model the lifetime of products because of its great flexibility). In the case of nanomedicines, it is not relevant to only quantify the concentration of the drug along time because it does not consider the stability of the NP. This was for example demonstrated in a work from Groo et al., in which the most effective paclitaxel nanoformulation on an in vivo subcutaneous tumor model after oral delivery were not those with the highest AUC. Importantly, the tumor was resistant to free paclitaxel and not to encapsulated paclitaxel. Thus, the formulation which was not stable during the absorption gave the highest AUC while free paclitaxel was not enough effective against the tumor. Paclitaxel encapsulated in stable LNCs was less absorbed but much more effective. It is one examples which demonstrates that relationship 
between AUC and drug effect is not obvious in the case of nanomedicines [38].

\section{Distribution}

The main goal of drug nanoencapsulation is to modify the distribution of the drug, for targeting purposes, in order to enhance its activity and/or to lower its toxicity. Thus, this step of the PK process should be clearly assessed. Once again, regular PK models fail to describe with good accuracy the dynamics of nanoparticle distribution, which depends on the interaction of particles with body fluids and tissues all along the distribution process. Physicochemical parameters of the NP are indeed changing after contact with body fluids and are responsible for the fate of the nanocarrier in vivo. To describe and predict those interactions it is mandatory to well characterize the NP before administration and during their journey in the bloodstream.

\section{Characterization of NP before administration}

To follow the distribution dynamics and to set-up relevant models, it is important to characterize the formulation before administration. Not only size, shape, and charge should be assessed, but the homogeneity of the formulation should be welldefined. If more than one type of nanostructure exists (for example nanocapsules mixed with micelles) in the same formulation mixture this will have an impact on the overall distribution. Regular PK models used to describe distribution are only considering the active ingredient as a free drug. Some nanomedicines PK models have been published but with the hypothesis that only one type of nanoparticle was administered [23], [39]. However, it is now well known that other species such as micelles can also be present in the formulation besides NP. This is also the case for the liposomal formulation, which can contain different types of liposomes or liposome remnants[40]. This complexity of the NP population after formulation has to be well characterized or purified in order to be included for a specific PK modeling of NP.

To explain the complexity of nanoparticle characterization we can look at an apparently simple parameter namely size determination. It is well known that size has an impact on distribution[41-42]. Thus, accurately determining the size is mandatory. However, different techniques adapted and validated in the nanosize range such as Dynamic Light Scattering (DLS) or Nanoparticle Tracking Analysis (NTA) or Field-Flow Fractionation (FFF) coupled to multiple sizing detectors or even Electron Microscopy (EM), will not give the same results especially for particles, which are not solid such as liposomes or lipid nanocapsules. That can appear normal because if the technique is different, the measured parameter is also different so the final result is different. In fact, the difficulty is to choose the best adapted method of analysis. Even more, size measurement is always performed in vitro in standardized conditions and this parameter may not be the same after contact with organic fluids as blood. The same reasoning can be applied for zeta potential reflecting the surface charge of the particles.

The access to the true value of size is thus difficult and impedes accurate modeling of distribution. A $10 \%$ error on the radius of the particle will lead to a $21 \%$ error on the surface and for some techniques, this difference is much higher [43].

\section{Characterization of NP after administration}

If nanoparticle characterization is not easy before administration while NP are in their native form, it becomes even much more difficult in the blood compartment after contact with plasma proteins, which forms a corona at the surfaces of the NP and potentially changes their diffusion properties [44], [45]. The profile of coating protein depends on the physicochemical properties of NP. This physicochemical specificity will lead to a "biological identity" which has been well described by Dawson and Linse et al., and more recently by Caracciolo et al. [14], [46]. This relative modern concept is misunderstood while mastering it, is probably one of the keys for nanomedicine clinical success [47]. Increasing knowledge of this phenomenon is essential to obtain information about surfaceassociated proteins of NP. Since the environment of protein changes between different body compartments, NP coating changes during their body traffic. Indeed, corona protein is constituted of "hard corona" based on strong NP/protein links, and "soft corona" due to a weak binding which allows dynamic protein exchanges at the surface of NP [48], [49]. Finally, protein corona formation can lead to opsonization which may enhance uptake by specialized cells of the reticuloendothelial system (RES) leading to an increase of NP elimination. Therefore, by changing the size, shape properties, and surface charge of NP, protein coating will influence NP biodistribution. To avoid or minimize corona protein and thus increase longer plasma half-life during biodistribution time, it is possible to design stealth NP with polyethylene ethylene glycol (PEG) covering their surface[50-51]. These NP could be considered as the second generation of NP for which a steric stabilization with PEG is one of the promising approaches employed to improve the PK profile, increase circulation time, 
or stability and reduce the clearance of plasma nanocarrier by RES system. [52]

Another important information to obtain to better set-up the PK model is the dynamic of equilibrium between the encapsulated drug, the free drug, and the free nanoparticle. The regular PK models only describe the evolution of drug concentrations in compartments such as blood, brain, or others specific tissues. In the case of NP, its performance is linked to the distribution process of the drug within the carrier (specific targeting or drug protection) and the kinetics of release from the carrier to obtain drug action. Thus, monitoring the drug alone presents some limitations for NP PK modeling. In this goal, it is important to use some methods allowing the determination of the encapsulated drug, the released drug but also emptied NP. All that information, obtained along the PK process, is needed to set-up an accurate PK model with a high prediction capability of both efficacy and toxicity of nanomedicines.

Singh et al. have performed an in vivo PK study on liposomes loaded with exemestane (Figure 1), in which they only measure drug concentration without evaluating the integrity of liposomes [23] as well as in the study of Menzel et al. with exenatide loaded in a self-emulsifying drug delivered system [39]. Most of the published studies rely on the monitoring of drug bloodconcentration along time, which is relevant for non-encapsulated drugs but is not accurate for nanomedicines as stated before. To consider the equilibrium between the free and the encapsulated drug during PK modeling is a requirement to knock down the barriers that currently impair the clinical translatability of nanomedicine treatments.

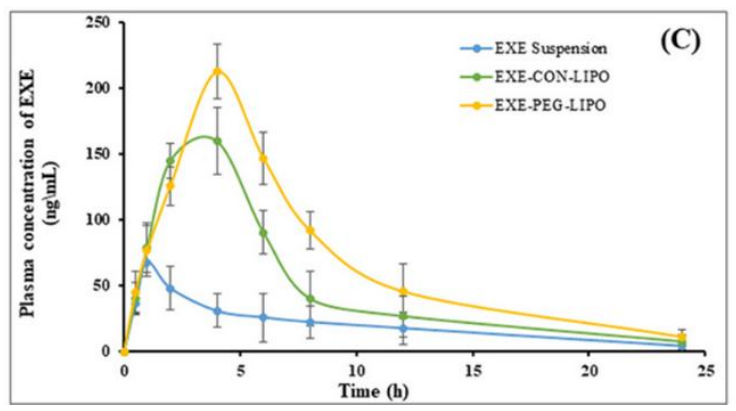

Figure 1 : Plasma concentration of exemestane (EXE) with time for three different formulations: suspension, conventional liposomes (EXE-CON-LIPO), and poly(ethylene glycol) (PEG)ylated liposomes (EXE-PEG-LIPO). Reproduced, with permission from 23]

Many studies use labelling of the drug or the nanocarrier (for example with a dye) to follow their distribution. In this case, the stability of the dye and of its association with the nanocarrier has to be checked. Many pitfalls have been reported like instability of fluorescent labelling, structure modification of the carrier after labelling, or stability deterioration of NP [53], [54]. Moreover, labelling can change the fate of the nanomedicines if it changes their surface properties as discussed before. Guo et al. give some details about each method of labelling and some flaws, for example, some elements of whole blood (haemoglobin) can interfere with fluorescence. According to this author, radiotracers are considered as a good choice to keep the molecular structure of the nanocarriers but unfortunately are not the best control of NP structure integrity [54].

\section{Volume of distribution}

The volume of distribution (Vd) is a theoretical volume where the drug should distribute at a homogenous concentration calculated from the initial blood concentration just after intravenous injection. This parameter is calculated for every regular drug product. In the case of nanomedicines, encapsulated and free drugs are present at the same time from the initial time point (TO), in the blood circulation because in many cases even in the containers there is an equilibrium between free and encapsulated drug. Because of this duality, the regular determination of $\mathrm{Vd}$ from the blood at TO is not accurate nor informative. If one calculates the $\mathrm{Vd}$ from the total drug (encapsulated plus free drug) the interpretation will be complex as both entities do not display the same diffusion patterns. The impossibility to determine an accurate $\mathrm{Vd}$ has some consequences on modeling because, in standard models, $\mathrm{Vd}$ is used to calculate other parameters such as drug total clearance. This example shows again that regular modeling is not relevant for PK determination of nanomedicines.

\section{Biodistribution studies}

One on the major way to evaluate the distribution of nanomedicines in the body while assessing their stability is to use FRET as already described for absorption studies. In this goal, Laine et al. used the FRET technique to evaluate the behavior of different lipid NP i.e LNC, Pegylated LNC, Lipid NanoEmulsions (LNEs). Results showed different release kinetic profiles, PEGylated LNCs and LNEs having an extended blood-circulation time associated with good structure stability over several hours after intravenous injection [55]. Gravier et al., have used macroscopic and microscopic imaging to analyze the FRET signal in order to determine the stability of LNE and LNC in a biological environment. Results showed that LNCs remained more stable than LNEs in presence of serum or cancer cells [56]. Those studies based only on imaging give some insight on the fate of 
the nanocarriers in vivo but cannot be quantitative to allow the determination of a PK profile. Quantifying both the free drug and the nanocarrier in parallel would be a good strategy in this aim.

Plenty of studies describe the surface modification of NPs to optimize the biodistribution of the encapsulated drug for example in active targeting on solid tumors. Some surface probes such as RGD peptides [57] or specific antibodies have been proposed in the past [11], [58]. In recent years, the ability of blood proteins to form a corona around the NP have also been taken into account to develop efficient active targeting [59-60]. Before trying to optimize the biodistribution, one should be able to accurately characterize the distribution of the nanomedicines and to differentiate the encapsulated drug from the free drug in every tissue. This would help to solve the issue of active targeting. In fact, the percentage of administrated drug which is considered to reach its target after encapsulation in specialized drug delivery systems is low and found between $0.3 \%$ to $3 \%$ according to some studies [61], [62]. Targeting has been one of the potential key features raised for nanomedicines, it is now obvious that this goal is not achieved in humans. This is also because animal models used (i.e mainly rodents) do not display the same physiological characteristics than humans and were thus non relevant models. It is also very difficult to modelize targeting.

\section{Conclusion on PK model relevancy for distribution step}

To characterize and modelize the distribution, it is important to determine the dynamics of corona formation over the particles. Regular PK parameters are not directly usable: as discussed before, this is for example the case of $\mathrm{Vd}$. Moreover, modeling should include the equilibrium between free and encapsulated drug, one way of doing this would be to add a supplemental compartment related to the encapsulated drug in the PK model. The minimum number of compartments in a nanomedicine PK model would thus be 2 i.e free drug in the blood and encapsulated drug in the blood (Figure 2).

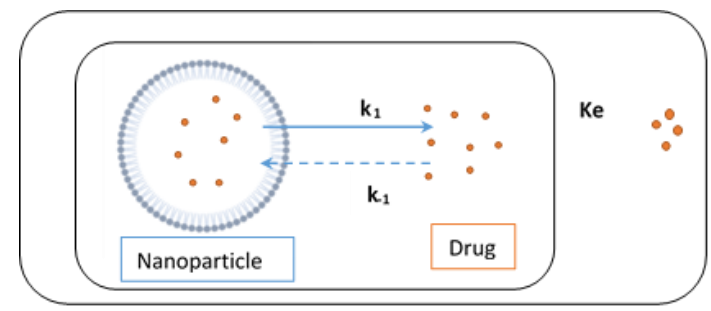

Figure 2: Drug release and exchange between nanoparticles and its elimination from the central compartment. $K 1$ and $K-1$ are the exchange constants of active drug between the NP central compartment. Ke represents the elimination constant from the compartment.

\section{Degradation - Elimination}

This phase is the latest phase of the PK process for drugs and NPs. One of the key features of NPs, which has been raised in the past to put forward those delivery systems is the prolonged circulation time. This has been seen as particularly relevant in the case of cancer therapy [5]. This is why it is really important to characterize this phase in view of optimizing it. This phase contains metabolization and excretion processes.

\section{Phases of NP elimination and hepatic affinity}

In the following, we will first consider a regular PK process related to the active drug (the cargo) and then what is related to the NP (the carrier).

In the case of the active drug, the PK phase of metabolism is mainly represented by chemical reactions mainly found in the liver. Usually, these reactions are divided into three phases. First, an activation phase using enzymes of cytochrome P450, which include oxidation, reduction, or hydrolysis reactions. Then, a conjugation phase is necessary because metabolites obtained after the first phase are clearly unstable. During this second phase, the addition of a hydrophilic group allows the best drug solubilization and thus facilitates its elimination. The last and third phase is defined as a phase of transport to eliminate xenobiotics [63] (Figure 3). All these phases refer to the active drug and not to its carrier.

In the case of nanomedicines, it seems obvious that these PK parameters associated with the usual metabolism and followed by drug elimination, are not directly applicable. As presented in Figure 3, for the nanocarrier, it could be possible to define a Phase IV of degradation. Indeed, even if NPs are not metabolized using the classical hepatic pathways, there is a specific process for NP degradation mainly linked to liver-NP interactions as described by Zhang et al. [64]. After NP administration, as it was already presented, proteins that are present in different biological media cover NPs to form a protein corona. This step called opsonization can be considered as the first step of elimination during which the NP is tagged by proteins to allow its recognition by specific cells. Indeed, NPs covered with this protein corona generate different cell interactions in the blood such as complement activation or in different tissues for example in the liver with cellular uptake by resident macrophages, the Kupffer cells. The second step of NP elimination is the result of these interactions which lead to a degradation of NP by the immune system. It clearly appears that the liver plays an essential role in the 
elimination of NP especially in the Kupffer cells by micropinocytosis, clathrin-mediated endocytosis or caveolin-mediated endocytosis [64-66]. Internalization is largely associated with surface charge, shape, or size and thus also associated with composition of protein corona because it has an impact on those three characteristics. For example, PEG reduces cellular uptake by decreasing the formation of protein corona. On the other hand, the NP size can affect endocytosis mechanisms. Indeed, endocytosis is caveolin-mediated for 20$100 \mathrm{~nm}$ NPs, whereas it is clathrin-mediated for 100-350 nm NPs, and it uses micropinocytosis for spleen, and lymph nodes [69]. It is one of the most important barriers to NP therapeutic efficiency [70]. PEG is added on NP surface to reduce uptake by MPS, but this can induce production of anti-PEG antibodies and activation of complement proteins. This mechanism of immunogenicity, which leads to an accelerated blood clearance $(A B C)$ phenomenon [71], is well established in preclinical studies but always unclear on the clinical situation [68], [7173]. The lack of relevant PK studies including repetition of NP injections could explain this situation. In the future, it will be interesting to study more precisely this $A B C$ phenomenon, especially PK modification of nanomedicines after

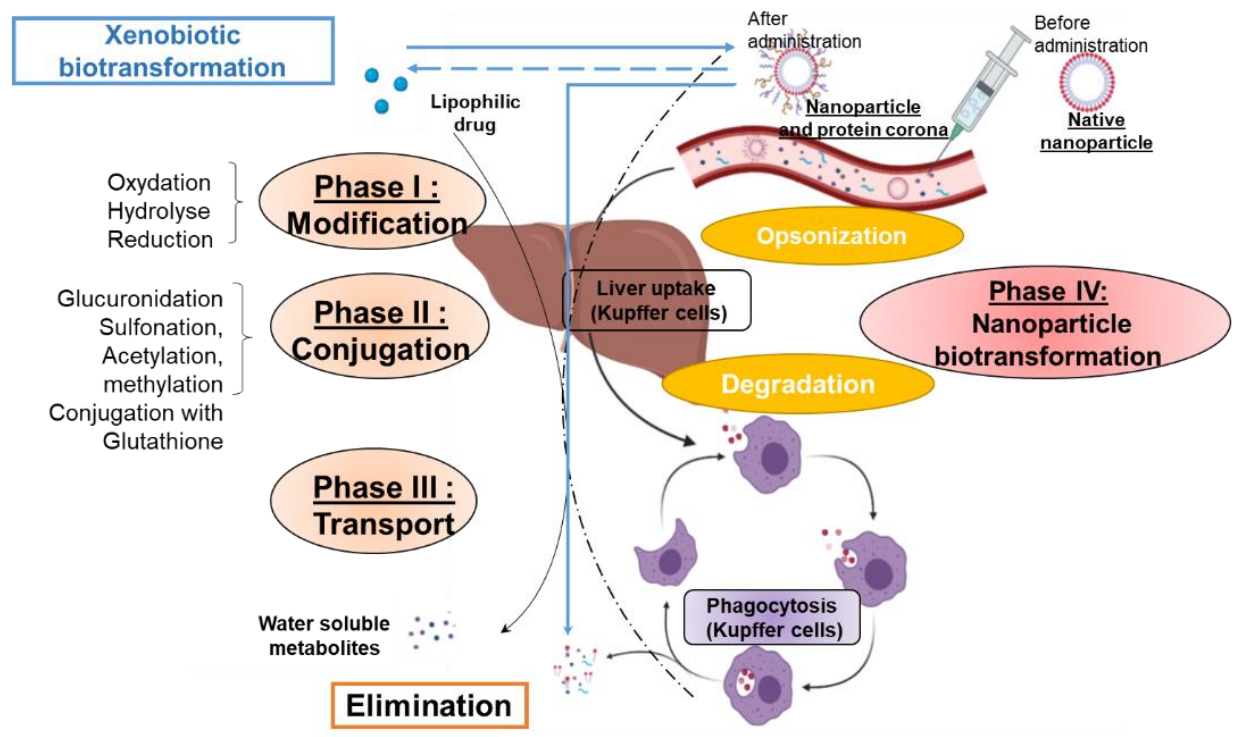

Figure 3: Parallel elimination of organic nanoparticles (a) and its encapsulated drug after release (b). Blue arrow represents drug release into the environment from the carrier; dashed blue arrow indicates drug exchange between the environment and the carrier. Blue circles indicate lipophilic drug molecules.

larger NPs [65-68].

\section{Nanoparticle liver interactions - ABC phenomena}

NP liver interactions is a major step of elimination that, unfortunately, was generally only studied in vitro or in healthy animals as stated by La-Beck et al.[69]. Using the right models in this case can be particularly relevant. For example, cancer diseases are often associated with profound and heterogeneous immune dysfunction, which can lead to modification of circulating proteins; thus changing the nature of the corona covering the NP and modifying their PK profiles [68]. In this respect it is worth to note that phagocytosis of NPs is more associated with specific recognizable proteins present on their surface rather than the quantity of binding proteins. Indeed, according to the study of Nguyen et al., uptake of NP in different protein concentrations mixtures by phagocytes was identical [60].

These interactions with the mononuclear phagocyte system (MPS) lead to NP clearance from blood and sequestration in organs of MPS: liver, multiple injections. $A B C$ phenomenon is complex and depends on multiple factors: time interval effect (more pronounced from 4 to 7 days), third dose effect (decrease of effect after two injections), PEG-surface density, PEG-chain-length effect, NP size and charge effect (increased for larger and positively charged NPs), administration effect (more important for slow infusion in contrast to bolus) or also animal species effect (maximum of $A B C$ is observed after 7 days for the rats, 10 days for the mice but unobserved for the rabbit) [71].

\section{Renal excretion}

As discussed for the distribution phase, a way to improve the PK profile on the elimination phase consists to adding PEG on the NP surface in order to reduce renal clearance [74].

NP renal elimination is also associated with protein-corona formation as it plays a major role in increasing particle diameter (hydrodynamic diameter) which becomes considerably larger as compared to its in vitro measurement. 
Consequences on blood clearance are important because NP diameter is associated with cut-off glomerular filtration linked to the elimination of the drug still being encapsulated from blood, so this can affect its blood half-life [75].

Glomerular tissue is composed of three layers: fenestrated endothelium, glomerular basement membrane (GBM), and podocytes with pores between $4-5 \mathrm{~nm}$. Filtration cut-off is around 65 $\mathrm{kDa}$ (albumin molecular weight), which corresponds to size from 6 to $8 \mathrm{~nm}$. NP measuring less than $6 \mathrm{~nm}$ are subject to free glomerular filtration while NP with a size upper than $8 \mathrm{~nm}$ are not filtered. Moreover, renal elimination is also influenced by NP charge because the glomerular basement membrane is negatively charged, so positively charged NPs are easier filtered than negatively charged NPs which are repulsed. In addition, because fenestrated endothelium is covered by a negatively charged glycocalyx layer of 200-300 nm thickness, it allows negative NPs to have a longer circulation time [54], [75- 76]. In a recent review, Peng et al. discussed the challenge of renal clearable nanocarriers. This is an interesting topic because the design of renal clearable nanomedicines have many advantages such as minimizing MPS uptake, and avoiding longterm accumulation in vital organs which could induce hepatic or spleen toxicity [70].

\section{Calculating PK parameters for elimination phase in the case of nanomedicines}

In regular PK analysis, the elimination phase is described by different PK parameters: half-life, mean retention time (MRT) and clearance. Those parameters are calculated from concentration of the free drug in the blood (mainly) versus time. This can be done by non-compartmental analysis (NCA) or after using compartmental modeling. In every case, the calculation should be related to the drug concentration. When it comes to nanomedicine, the problem is much more complex as the drug can be present in blood as free drug or still being encapsulated (i.e. not bioavailable to the site of action). This particular situation should be taken into account in studies involving PK analysis of nanomedicines. Indeed, the obtained result is not the same if both the free drug and the encapsulated one are separated after blood sampling before analysis. Because the fraction of drug that can be eliminated by the kidney is only the free drug, the calculated renal clearance should only refer to the free drug (with exception of clearable nanomedicines with a size below $8 \mathrm{~nm}$ as discussed before). For terminal half-life determination, the issue is the same and interpreting a half-life that includes free and encapsulated drug may be very tricky especially if the parameter is compared with a standard injectable or oral formulation without any encapsulation i.e. sequestration of the drug from its elimination process. In this aspect, the MRT may be much more relevant as it allows a fair comparison between different formulations of the same drug after non-compartmental analysis. MRT is a global value of the meantime a molecule will stay in the studied tissue or media, it is only calculated from the area under the blood concentration curve (AUC) and its shape described by area under the first moment curve (AUMC). Total clearance calculation in the case of nanomedicine should also only be calculated as the ratio of dose and AUC using NCA once again by keeping in mind that in the case of nanomedicine the result will be different if free drug is considered or if total of free and encapsulated drug is considered. If modeling is involved to determine those elimination parameters, the carrier with its encapsulated drug should be considered as a supplementary compartment as discussed before. In the model, the stability of the carrier should also be considered as it has an impact on the release of the free drug and thus on its elimination. In fact, the released drug can follow the elimination process, which may not be the same for the encapsulated drug still protected from the major elimination processes (metabolization, kidney filtration...).

\section{Conclusion and perspectives}

Classical PK parameters are not fully adapted and thus classical PK modeling is not sufficient to describe the PK of nanomedicines. Indeed, we can conclude that the PK of NPs is a suite of complex phenomena which associate free drug, encapsulated drug, and empty NPs. PK classical models allow to describe only the fate of the free drug. It is now necessary to create new PK models taking into account the dynamic equilibrium of free and encapsulated drug, the stability of the nanocarrier in the organisms, its interactions with specific organs such as the liver, the spleen and the kidney and the in vivo modification of the carrier. Recently, Colino et al., proposed an interesting PK model of NP, which includes a specific compartment for macrophage uptake and release [66]. Then, two recent reviews written by Byun et al., and Yuan et al., gave some details about PK behavior to design PBPK models [77-78]. Finally, Guo et al., gave good propositions to improve PK modeling with two methods of data approach, compartmental and noncompartmental analysis. The second method is more robust especially when the distribution is unknown [54]. 


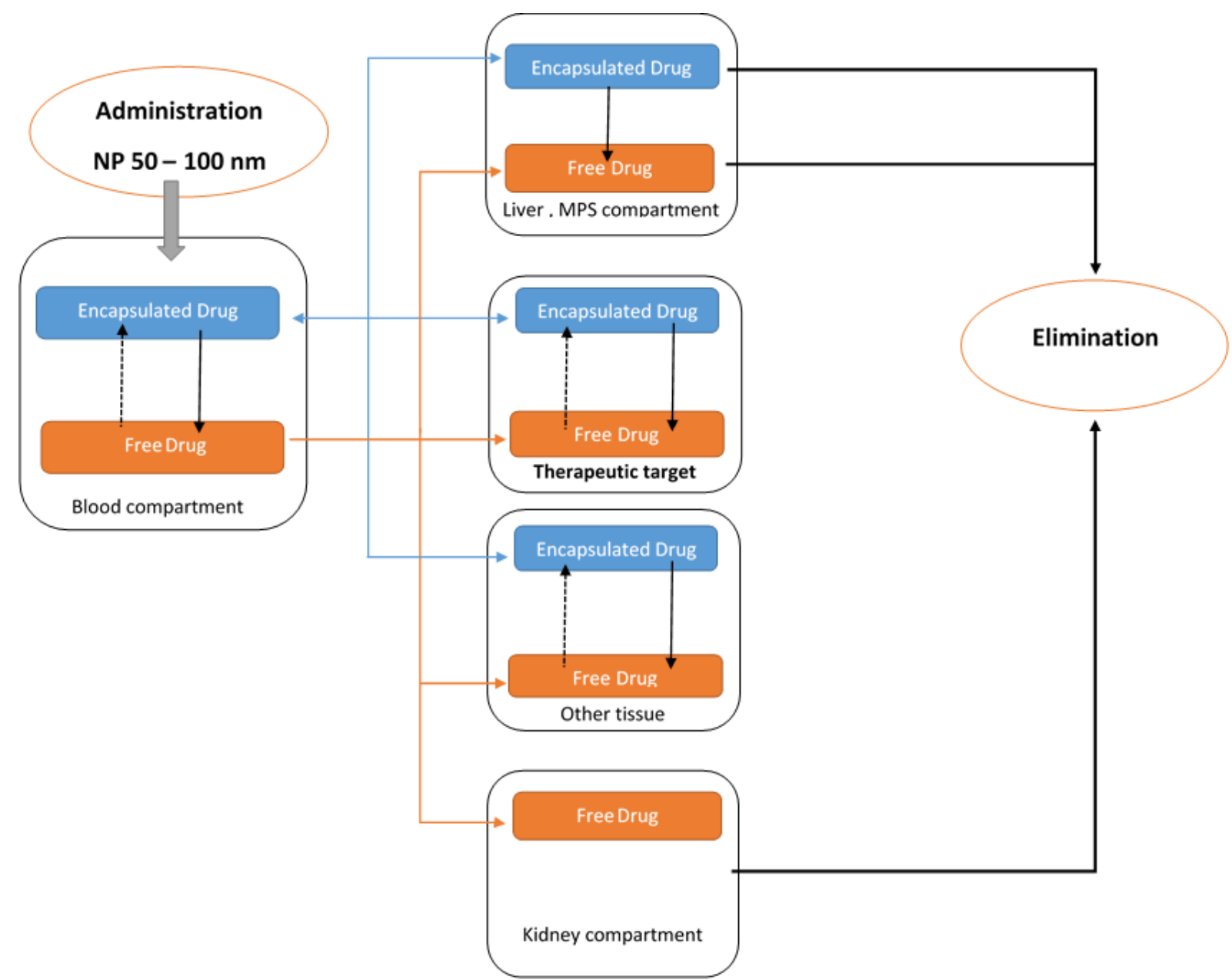

As these authors explain, it seems essential to take into account all sequestration compartments in mathematical models to better describe bloodcirculation time and elimination of nanomedicines. Figure 4 sums-up a tentative approach to describe the compartment to consider in modeling, which is the first step to relevant future PK models for nanomedicines.

\section{Acknowledgement}

Figure 4 : Proposed theoretical compartment pharmacokinetics (PK) model adapted to the specific features of nanomedicines. Blue arrows indicate encapsulated drug biodistribution; orange arrows indicate free drug biodistribution; black arrows indicate drug release from the nanoparticle; dashed black arrows indicate hypothetical return of drug back its carrier. Abbreviation: MPS, mononuclear phagocyte system.
This work was funded by the French league against cancer ("La Ligue contre le cancer") Maine et Loire Comity. 


\section{References}

1. Tran, S., DeGiovanni, P.-J., Piel, B. \& Rai, P. Cancer nanomedicine: a review of recent success in drug delivery. Clin. Transl. Med. Dec 11;6 (1):44 (2017).

2. Baden, L. R. et al. Efficacy and Safety of the mRNA-1273 SARS-CoV-2 Vaccine. N. Engl. J. Med. Feb 4;384(5):403-416 (2020).

3. Machhi, J. et al. Nanocarrier Vaccines for SARSCoV-2. Adv. Drug Deliv. Rev. Jan 8:S0169409x(21)00002-8 (2021).

4. Nanomedicine and the COVID-19 vaccines. Nature Nanotechnology. Dec;15(12):963. (2020).

5. Park, K. Drug delivery of the future: Chasing the invisible gorilla. J. Control. Release 240, 2-8 (2016).

6. Florence, A. T. Nanotechnologies for site specific drug delivery: Changing the narrative. International Journal of Pharmaceutics vol. 551 1-7 (2018).

7. Malhaire, H. \& Lagarce, F. Is the translational approach becoming a reality in nanomedicine? Eur. J. Nanomedicine vol. 7(2) 79-83 (2015).

8. Allen, T. M. \& Cullis, P. R. Drug Delivery Systems: Entering the Mainstream. Science. vol. 303 18181822 (2004).

9. Farjadian, F. et al. Nanopharmaceuticals and nanomedicines currently on the market: Challenges and opportunities. Nanomedicine. vol. 14 93-126 (2019).

10. Gabizon, A., Shmeeda, H. \& Barenholz, Y. Pharmacokinetics of Pegylated Liposomal Doxorubicin Review of Animal and Human Studies. Clin Pharmacokinet vol. 42(5):419-36. (2003).

11. Jain, R. K. \& Stylianopoulos, T. Delivering nanomedicine to solid tumors. Nature Reviews Clinical Oncology vol. 7 653-664 (2010).

12. Gradishar, W. J. et al. Phase III trial of nanoparticle albumin-bound paclitaxel compared with polyethylated castor oil-based paclitaxel in women with breast cancer. J. Clin. Oncol. 23, 77947803 (2005).

13. Danhier, F. "To exploit the tumor microenvironment: Since the EPR effect fails in the clinic, what is the future of nanomedicine?" $J$. Control. Release Dec 28;244(Pt A):108-121. (2016)

14. Caracciolo, G., Farokhzad, O. C. \& Mahmoudi, M. Biological Identity of Nanoparticles In Vivo: Clinical Implications of the Protein Corona. Trends in Biotechnology vol. 35 257-264 (2017).

15. Auffan, M. et al. Towards a definition of inorganic nanoparticles from an environmental, health and safety perspective. Nat. Nanotechnol. Oct;4(10):634-41., (2009).

16. Kalarikkal, N. \& Thomas, S. Challenges in nonparenteral nanomedicine therapy. in Theory and Applications of Nonparenteral Nanomedicines.
Kesharwani P., Taurin S., Greish K. (Eds) (pp 27-54) Elsevier (2021).

17. Malhaire, H., et al. How to design the surface of peptide-loaded nanoparticles for efficient oral bioavailability? Advanced Drug Delivery Reviews. Nov 15;106(Pt B):320-336. (2016).

18. Roger, E., Lagarce, F., Garcion, E. \& Benoit, J. P. Biopharmaceutical parameters to consider in order to alter the fate of nanocarriers after oral delivery. Nanomedicine. vol. 5 287-306 (2010).

19. Ramadan, A. et al. Oral fondaparinux: use of lipid nanocapsules as nanocarriers and in vivo pharmacokinetic study. Int. J. Nanomedicine. vol. 6:2941-51 (2011).

20. Varshosaz, J., Taymouri, S., JahanianNajafabadi, A. \& Alizadeh, A. Efavirenz oral delivery via lipid nanocapsules: formulation, optimisation, and ex-vivo gut permeation study. IET Nanobiotechnology. Sep;12(6):795-806., (2018).

21. Roger, E., Lagarce, F., Garcion, E. \& Benoit, J. Lipid nanocarriers improve paclitaxel transport throughout human intestinal. J. Control. Release. Dec 3;140(2):174-81 (2009).

22. Roger, E., Lagarce, F. \& Benoit, J. P. The gastrointestinal stability of lipid nanocapsules. Int. J. Pharm. 379, 260-265 (2009).

23. Singh, A., Neupane, Y. R., Shafi, S., Mangla, B. \& Kohli, K. PEGylated liposomes as an emerging therapeutic platform for oral nanomedicine in cancer therapy: in vitro and in vivo assessment. J. Mol. Liq. 303 (2020).

24. Tobío, M. et al. The role of PEG on the stability in digestive fluids and in vivo fate of PEG-PLA nanoparticles following oral administration. Colloids and Surfaces B: Biointerfaces. Oct 1;18(34):315-323. (2000).

25. Cone, R. A. Barrier properties of mucus. Advanced Drug Delivery Reviews vol. 61 75-85 (2009).

26. Groo, A. C. \& Lagarce, F. Mucus models to evaluate nanomedicines for diffusion. Drug Discovery Today vol. 19 1097-1108 (2014).

27. Sardelli, L. et al. Towards bioinspired: In vitro models of intestinal mucus. RSC Advances vol. 9 15887-15899 (2019).

28. Groo, A. C. et al. Development of 2D and 3D mucus models and their interactions with mucuspenetrating paclitaxel-loaded lipid nanocapsules. Pharm. Res. 31, 1753-1765 (2014).

29. Dünnhaupt, S. et al. A. Nano-carrier systems: Strategies to overcome the mucus gel barrier. European Journal of Pharmaceutics and Biopharmaceutics vol. 96 447-453 (2015).

30. des Rieux, A. et al. An improved in vitro model of human intestinal follicle-associated epithelium to study nanoparticle transport by M cells. Eur. J. Pharm. Sci. vol. 30, 380-391 (2007). 
31. Xu, Y., Shrestha, N., Préat, V. \& Beloqui, A. Overcoming the intestinal barrier: $A$ look into targeting approaches for improved oral drug delivery systems. J. Control. Release vol. 322, 486508 (2020).

32. Beloqui, A., des Rieux, A. \& Préat, V. Mechanisms of transport of polymeric and lipidic nanoparticles across the intestinal barrier. Advanced Drug Delivery Reviews vol. 106 242-255 (2016).

33. Pereira, C. et al. Dissecting stromal-epithelial interactions in a 3D in-vitro cellularized intestinal model for permeability studies. Biomaterials vol. 56, 36-45 (2015).

34. He, C., Yin, L., Tang, C. \& Yin, C. Size-dependent absorption mechanism of polymeric nanoparticles for oral delivery of protein drugs. Biomaterials vol. 33, 8569-8578 (2012).

35. Norris, D. A. \& Sinko, P. J. Effect of size, surface charge, and hydrophobicity on the translocation of polystyrene microspheres through gastrointestinal mucin. J. Appl. Polym. Sci. vol. 63(11), 1481-1492 (1997).

36. Roger, E. et al. Lipid nanocapsules maintain full integrity after crossing a human intestinal epithelium model. J. Control. Release. 253, 11-18 (2017).

37. Gullberg, E. et al. Expression of specific markers and particle transport in a new human intestinal M-cell model. Biochem. Biophys. Res. Commun. 279, 808-813 (2000).

38. Groo, A. C. et al. In vivo evaluation of paclitaxel-loaded lipid nanocapsules after intravenous and oral administration on resistant tumor. Nanomedicine. Mar;10(4):589-601. (2015).

39. Menzel, C. et al. In vivo evaluation of an oral self-emulsifying drug delivery system (SEDDS) for exenatide. J. Control. Release 277, 165-172 (2018). 40. He, H. et al. Adapting liposomes for oral drug delivery. Acta Pharmaceutica Sinica B vol. 9 36-48 (2019).

41. Partikel, K. et al. Effect of nanoparticle size and PEGylation on the protein corona of PLGA nanoparticles. Eur. J. Pharm. Biopharm. 141, 70-80 (2019).

42. Fan, W. et al. Effect of particle size on the pharmacokinetics and biodistribution of parenteral nanoemulsions. Int. J. Pharm. 30;586:119551. (2020).

43. Caputo, F., et al. Measuring particle size distribution of nanoparticle enabled medicinal products, the joint view of EUNCL and NCI-NCL. A step by step approach combining orthogonal measurements with increasing complexity. J. Control. Release. vol. 299, 31-43 (2019).

44. Capriotti, A. L., Cavaliere, C. \& Piovesana, S. Liposome protein corona characterization as a new approach in nanomedicine. Analytical and Bioanalytical Chemistry vol. 411, 4313-4326 (2019).

45. Ke, P. C., Lin, S., Parak, W. J., Davis, T. P. \& Caruso, F. A Decade of the Protein Corona. ACS Nano vol. 11, 11773-11776 (2017).

46. Cedervall, $\mathrm{T}$. et al. Understanding the nanoparticle-protein corona using methods to quantify exchange rates and affinities of proteins for nanoparticles. Proc Natl Acad Sci U S A, vol. 104(7), 2050-5 (2007).

47. Jain, P. et al. In-vitro in-vivo correlation (IVIVC) in nanomedicine: Is protein corona the missing link? Biotechnology Advances. vol. 35 889-904 (2017).

48. Kristensen, K. et al. The hard protein corona of stealth liposomes is sparse. J. Control. Release 307, 1-15 (2019).

49. Vilanova, O. et al. Understanding the Kinetics of Protein-Nanoparticle Corona Formation. ACS Nano 10, 10842-10850 (2016).

50. Palchetti, S. et al. Influence of dynamic flow environment on nanoparticle-protein corona: From protein patterns to uptake in cancer cells. Colloids Surfaces B Biointerfaces 153, 263-271 (2017).

51. Kari, O. K. et al. In situ analysis of liposome hard and soft protein corona structure and composition in a single label-free workflow. Nanoscale 12, 1728-1741 (2020).

52. Fang, G., et al. Nanomedicines for improved targetability to inflamed synovium for treatment of rheumatoid arthritis: Multi-functionalization as an emerging strategy to optimize therapeutic efficacy. J. Control. Release, vol. 303, 181-208 (2019).

53. Bastiat, G. et al. A new tool to ensure the fluorescent dye labeling stability of nanocarriers: A real challenge for fluorescence imaging. J. Control. Release. 28;170(3):334-42 (2013).

54. Guo, H. \& Mackay, J. A. A pharmacokinetics primer for preclinical nanomedicine research. in Nanoparticles for Biomedical Applications, $1^{\text {st }}$ Ed. Eun J. C., Lorraine L. \& RInaldi C. (Eds), Fundamental Concepts, Biological Interactions and Clinical Applications (pp. 109-128). Elsevier (2020).

55. Lainé, A. L. et al. Conventional versus stealth lipid nanoparticles: Formulation and in vivo fate prediction through FRET monitoring. J. Control. Release. 28;188:1-8. (2014).

56. Gravier, J. et al. FRET imaging approaches for in vitro and in vivo characterization of synthetic lipid nanoparticles. Mol. Pharm. 2. 11(9), 3133-44 (2014).

57. Goutayer, M. et al. Tumor targeting of functionalized lipid nanoparticles: Assessment by in vivo fluorescence imaging. Eur. J. Pharm. Biopharm. 75, 137-147 (2010). 
58. Hadjidemetriou, M. et al. In Vivo Biomolecule Corona around Blood-Circulating, Clinically Used and Antibody-Targeted Lipid Bilayer Nanoscale Vesicles. ACS Nano 9, 8142-8156 (2015).

59. Elechalawar, C. K. et al. Analysing the nanoparticle-protein corona for potential molecular target identification. Journal of Controlled Release. vol. 322, 122-136 (2020).

60. Nguyen, V. H. \& Lee, B. J. Protein corona: A new approach for nanomedicine design. International Journal of Nanomedicine vol. 12 3137-3151 (2017).

61. Dai, Q. et al. Quantifying the Ligand-Coated Nanoparticle Delivery to Cancer Cells in Solid Tumors. ACS Nano 12, 8423-8435 (2018).

62. S. Wilhelm et al., "Analysis of nanoparticle delivery to tumours," Nature Reviews Materials, vol. 1., 16014 (2016)

63. Xu, C., Li, C. Y.-T. \& Kong, A.-N. T. Induction of phase I, II and III drug metabolism/transport by xenobiotics. Arch. Pharm. Res. 28(3):249-68. (2005).

64. Zhang, Y.-N. et al. Nanoparticle-liver interactions: Cellular uptake and hepatobiliary elimination. J. Control. Release. 240, 332-348 (2016).

65. Xiao, K. et al. The effect of surface charge on in vivo biodistribution of PEG-oligocholic acid based micellar nanoparticles. Biomaterials 32, 3435-3446 (2011).

66. Colino, C. I., Lanao, J. M. \& Gutierrez-Millan, C. Targeting of Hepatic Macrophages by Therapeutic Nanoparticles. Frontiers in Immunology vol. 11:218 (2020).

67. Chan, W. C. W. Nanoparticle Size and Surface Chemistry Determine Serum Protein Adsorption and Macrophage Uptake. J Am Chem Soc. vol. 1;134(4):2139-47 (2012).

68. Mcgoron, A. et al. Harnessing Liposome Interactions With the Immune System for the Next Breakthrough in Cancer Drug Delivery. Front Pharmacol. vol. 12;10:220 (2019).

69. La-beck, N. M. \& Gabizon, A. A. Nanoparticle interactions with the immune system: clinical implications for Liposome-Based cancer chemotherapy. Front Immunol. vol. 8, 6-11 (2017). 70. Peng, C., Huang, Y. \& Zheng, J. Renal clearable nanocarriers: Overcoming the physiological barriers for precise drug delivery and clearance. J. Control. Release 322, 64-80 (2020).

71. Shimizu, T., et al. Relationship between the Concentration of Anti-polyethylene Glycol (PEG) Immunoglobulin $\mathrm{M}$ (IgM) and the Intensity of the Accelerated Blood Clearance ( $A B C$ ) Phenomenon against PEGylated Liposomes in Mice. Biol. Pharm. Bull. Pharm. Bull. vol. 38(3):417-24. (2015).

72. Armstrong, J. K. et al. Antibody against poly(ethylene glycol) adversely affects PEGasparaginase therapy in acute lymphoblastic leukemia patients. Cancer 110, 103-111 (2007).

73. Zhang, Z. et al. Anti-PEG scFv corona ameliorates accelerated blood clearance phenomenon of PEGylated nanomedicines. J. Control. Release. vol. 10, 330:493-501. (2020).

74. Alexis, F., Pridgen, E., Molnar, L. K. \& Farokhzad, O. C. Factors affecting the clearance and biodistribution of polymeric nanoparticles. Mol. Pharm. 5, 505-15 (2008).

75. Longmire, M., Choyke, P. L. \& Kobayashi, H. Clearance properties of nano-sized particles and molecules as imaging agents: Considerations and caveats. Nanomedicine vol. 3 703-717 (2008).

76. Bertrand, N. \& Leroux, J. C. The journey of a drug-carrier in the body: An anatomo-physiological perspective. J. Control. Release vol. 161 152-163 (2012).

77. Byun, J. H. et al. Recent advances in physiologically based pharmacokinetic and pharmacodynamic models for anticancer nanomedicines. Archives of Pharmacal Research vol. 43 80-99 (2020).

78. Yuan, D. et al. Physiologically Based Pharmacokinetic Modeling of Nanoparticles. J. Pharm. Sciences. vol. 108 58-72 (2019). 
